\title{
A Review on Performance Analysis of PDE Based Anisotropic Diffusion Approaches for Image Enhancement
}

\author{
Niveditta Thakur, Nafis Uddin Khan and Sunil Datt Sharma \\ Jaypee University of Information Technology, Solan, India \\ E-mail: niveditta.thakur06@gmail.com,nafisuddin.khan@juit.ac.in,sdsharma.juet@rediffmail.com
}

Keywords: image enhancement, image de-noising, anisotropic diffusion, edge preservation

Received: October 13, 2020

\begin{abstract}
Partial differential equation based anisotropic diffusion techniques are used extensively in computer vision for image enhancement and de-noising. Anisotropic diffusion is found to be an efficient and low computational complexity approach that has overcome the undesirable effects of linear smoothing filters and now is popular in prominent research areas of enhancing the quality of low contrast images and speckle noise reduction from geological, industrial, and medical images. This paper presents state-of-theart anisotropic diffusion technique and a comprehensive survey on various advancements in anisotropic diffusion for image enhancement and de-noising. The capability of anisotropic diffusion for enhancing the quality of low contrast images and speckle noise reduction from medical and industrial images are further explored. Various quality measures used to validate the performance are studied. The major research issues and possible future scopes in anisotropic diffusion filtering are also discussed.
\end{abstract}

Povzetek: Prispevek predstavi pregled in novo metodo na področju anizotropne difuzije za povečevanje slik in zmanjševanje šuma.

\section{Introduction}

Image quality improvement along with removal of noise and de-blurring are the prime objectives in the field of image processing. Image enhancement and de-noising [1] are vital topics in image processing literature where the techniques used to remove these imperfections from the images are discussed. The images carrying useful information needs to get enhance for proper visualization. However, the adverse external and environmental conditions at the time of image capturing and poor quality of imaging devices are some of the principal causes of noise [2] in images. Speckle noise and Gaussian blurring are dominant in medical images, remote sensing and computer tomography images. Magnetic resonance imaging (MRI) is affected by Rician and Poisson's noise. Poor contrast of medical images is also a serious problem in medical diagnosis and modality. Therefore, image enhancement and de-noising has now become a very popular and challenging issue in extracting the useful information from all the different types of images. In earlier days, the linear filtering approaches were used to deal with such noises in image. In the linear filtering approach, the pixel values are replaced by linear combinations of the pixels around the test pixel in a predefined neighborhood [1]. Linear filters perform well in denoising but not good at edge points and fine details where they tend to blur the edges and fail to protect the image information. Spatial nonlinear filters reduce noise without edge blurs and thus preserve the edges. But, the problem is to recover the meaningful edges in a coarse image under the presence of noise and poor contrast. The anisotropic diffusion approaches are being used in image processing since 1987 when Perona and Malik [3] have introduced a non-linear method of edge preserving smoothing that outperformed the existing traditional linear methods. Anisotropic diffusion [3] also referred to as nonlinear diffusion or Perona-Malik diffusion (PMD) is state-of-theart technique aims at smoothing noise from the image along with edge and fine detail preservation. This is originated from the process that creates a scale of more and more smoothed images which correspond to the result of convolution of the image with a 2D isotropic Gaussian filter. The width of the Gaussian filter is made to vary with the scale parameter which is analogous to linear and space-invariant transformation of the original image and thus called as iso-tropic or linear diffusion. However, anisotropic diffusion as given by Perona and Malik diffusion (PMD) [3] is a generalized improvement of the linear diffusion process where the filter was made to depend on the local content of the original image. The convolution between the original image and contentdependent filter produces a scale of smoothed images along with the preservation of essential contents like edges, lines, curves, and boundaries. Consequently, anisotropic diffusion acts as a non-linear and space-variant transformation of the original image which is now a very common image enhancement and de-noising tool in this area. This paper presents a comprehensive study of various anisotropic diffusion techniques developed so far and their analysis in concern with speckle noise reduction and contrast improvement of medical and industrial images.

The rest of the paper is organized as follows. Section 2 describes the origin and critics of basic anisotropic diffusion filtering in images. A comprehensive survey on 
the advancement of anisotropic diffusion in the field of contrast enhancement and speckle noise reduction has been presented in Section 3. Various image quality assessment parameters used for validation of anisotropic diffusion methods are mentioned in Section 4. A brief discussion and observations for future research exploration are given in Section 5 and finally, the paper is concluded in Section 6.

\section{Origin of anisotropic diffusion}

The partial differential equation (PDE) based anisotropic diffusion is state-of-the-art image processing technique originated by a well-known mathematical formulation of Fick's law [4]. The physical observation of this law signifies the equilibrium property between the concentration differences without creating or destroying the mass. This law states that a concentration gradient generates a flux which tries to compensate this gradient. The expression of Fick's law with continuity equation gives rise to the linear diffusion process which is also known as isotropic diffusion defined below:

$\frac{d I_{t}}{d t}=\operatorname{div}(C . \nabla I)$

where I is input image, $\nabla \mathrm{I}$ is image gradient, $\mathrm{t}$ is iteration and $\mathrm{C}$ is diffusion coefficient which is kept constant for the entire image. This diffusion equation in image corresponds to smoothing which is just analogous to the linear convolution of image with a Gaussian smoothing filter of standard deviation equal to $\sqrt{2} \mathrm{t}$. This tends to smooth the entire image equally in all directions without taking care of edges, boundaries and fine details. This demerit of linear diffusion filtering is solved by introducing the new diffusion coefficient [3] which depends on the image structures. This idea of adaptivity in the diffusion process give rise to anisotropic diffusion (PMD)[3] which is as shown below:

$\frac{d I_{t}}{d t}=\operatorname{div}(C(\nabla I) \cdot \nabla I)$

where $C(\nabla I)$ is now termed as diffusion coefficient function which is given as:

$$
C(\nabla I)=\frac{1}{1+\left(\frac{|\nabla I|}{k}\right)^{2}}
$$

The diffusion coefficient function denoted by $\mathrm{C}(\mathrm{\nabla I})$ in diffusion equation (Eq. (2)) controls the diffusion process by varying it in accordance with the gradient magnitude $\nabla \mathrm{I}$ at each pixel of the image. A fixed edge threshold parameter denoted by $\mathrm{k}$ is used depending on the type of the image. The diffusion in the images with high $\mathrm{k}$ values tends to strongly smooth the image whereas lower values of $\mathrm{k}$ are used to slow down the smoothing process near edges. This helps in the preservation of fine edges exhibiting high gradient magnitudes along with smoothing of low gradient homogeneous noisy background of the image.

\subsection{Critics in state of the art}

The anisotropic diffusion approach introduced by Perona and Malik (PMD) is quite effective in the images affected by Gaussian noise. However, the presence of impulse noise leads to image quality degradation. The impulse
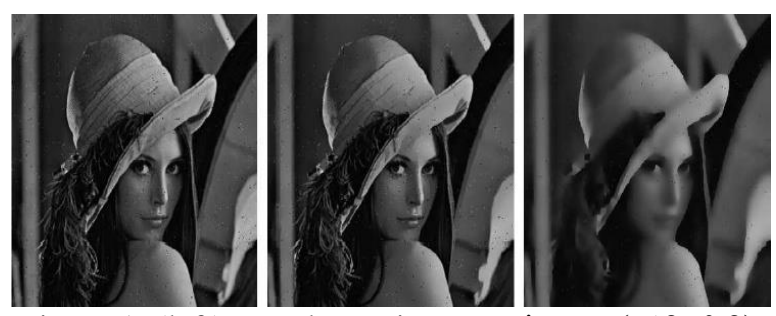

Figure 1: (left) Impulse Noisy Lena image $\left(\sigma^{\wedge} 2=0.2\right)$;

(middle) Restored image by anisotropic diffusion

$(\mathrm{k}=4, \mathrm{~T}=100)$; (right) Restored image by anisotropic diffusion $(\mathrm{k}=10, \mathrm{~T}=100)$.
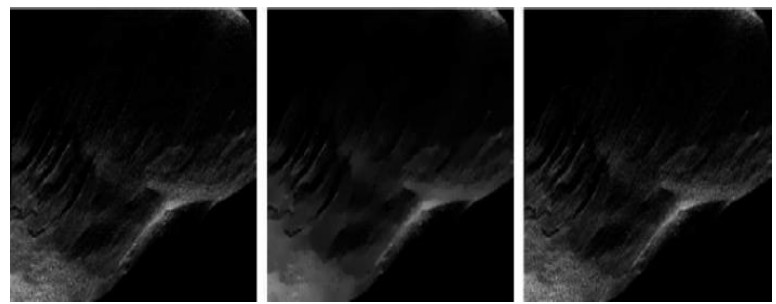

Figure 2: (left) Original low contrast moon surface image; (middle) Restored image by anisotropic diffusion $(\mathrm{k}=4, \mathrm{~T}=50)$; (right) Restored image by anisotropic diffusion $(\mathrm{k}=2, \mathrm{~T}=50)$.

noisy pixels in images due to high gradient magnitudes are treated as edge pixels by the diffusion process and get preserved in the restored images as shown in Figure 1. Here, a noisy 'Lena' image with noise variance $\sigma^{\wedge} 2=0.2$ has been restored by anisotropic diffusion by varying the value of $\mathrm{k}$. Such problems have been observed in medical images where speckles appear in the output image due to the diffusion process.

The consequences of PMD in low contrast images are quite unsatisfactory as the low gray level edges and the noisy background in such low contrast images are difficult to discriminate. The assumption used in PMD is that the gradient magnitude of edges is larger than that of the noisy back-ground which tends to enhance the corresponding edges at a particular value of edge threshold parameter $\mathrm{k}$. However, in low contrast images, the low gray level interregion edges with lower gradient magnitude can never be enhanced at the same edge threshold parameter. This defect has been demonstrated in Figure 2.

\section{Review on anisotropic diffusion}

The role of anisotropic diffusion filtering in enhancing a wide variety of distorted images has played an important role in medical, forensic, industrial, and military applications. The success of anisotropic diffusion based approaches is mainly dependent on the proper selection of diffusion coefficient function $\mathrm{C}(\nabla \mathrm{I})$ and the edge threshold parameter k. Efficient enhancement of noisy image follows causality, piecewise smoothing, and proper edge localization [3]. As presented by Perona and Malik, anisotropic diffusion equation (PMD) uses four nearest neighbors to calculate the gradient in diffusion coefficient function as given in Eq. (4):

$I_{t+1}=I_{t}+\frac{1}{4} \sum_{i=1}^{4}\left[C_{t} . \nabla I_{t}\right]$ 
The diffusion coefficient function $C(\nabla I)$ of Perona and Malik diffusion equation is replaced by $C\left(\nabla\left(G_{\sigma_{r}} * I\right)\right)$ as shown in Eq. (5) where the given image is initially convolved with a Gaussian kernel $G_{\sigma_{r}}$ of standard deviation $\sigma_{r}$ and then the gradient is calculated to get the resultant modified diffusion equation (CLMCD) [5].

$\frac{\partial I_{t}}{\partial t}=\operatorname{Div}\left[d\left(\left|\nabla\left(G_{\sigma_{r}} * I\right)\right|\right) \cdot \nabla I\right]$

This equation is undoubtedly capable of enhancing edges and all structures. However, there are strong blurring effects as the regularizing Gaussian kernel size $\sigma$ increases. You and Kaveh proposed the fourth-order PDE model (YKD) [6] for edge detection by using Laplacian $\nabla \mathrm{I}$ in place of gradient in diffusion coefficient function as shown below:

$C(|\Delta I|)=\frac{1}{1+\left(\frac{\mid \Delta I I}{k}\right)^{2}}$

The method was further extended by Chen et al. [7] where a coupled anisotropic diffusion (CBMD) with a new edge stopping function was developed which is as follows:

$\frac{\partial I_{t}}{\partial t}=v(t) \operatorname{Div} \frac{\left(\nabla\left(G_{\left.\sigma_{r} * I\right)}\right)\right.}{\left|\nabla\left(G_{\sigma_{r}} * I\right)\right|}-u\left(\left(G_{\sigma_{r}} * I\right)-I\right)$

where $\mathrm{v}(\mathrm{t})$ is a function of time and uis a constant. Weeratunga and Kamath [8] suggested the use of regularized version of image to calculate gradient as given in Eq. (8). This helps in converting ill-posed formulation of conventional anisotropic diffusion into well-posed problem and also make parameter $\mathrm{k}$ variable to facilitate the enhancement of edges with different gradients.

$\nabla I=\nabla\left(G_{\sigma_{r}} * I\right)$

A complex diffusion equation (GCD) was developed by Gilboa et al. [9] where the diffusion coefficient function represents a complex value with an imaginary part acts as an edge detector and the real part is responsible for ramp preserving de-noising. The complex diffusion coefficient function is given as:

$C\left(I_{m}(I)\right)=\frac{e^{j \theta}}{1+\left(\frac{I_{m}(I)}{k \theta}\right)^{2}}$

where I_m (I) is the imaginary value of the image Inormalized by phase angle $\theta$ which should be small $(\theta \ll 1)$ and $\mathrm{k}$ is the threshold parameter. When $\theta$ is small then it is similar to real diffusion and when it approaches to $90^{\wedge} 0$, the implementation of complex diffusion with incremental time-steps becomes an inefficient process. Tschumperle and Deriche [10] defined the Hessian matrices based anisotropic diffusion formulation (TDD) for vector-valued images which is expressed in Eq. (10).

$\frac{\partial I_{t}}{\partial t}=\sum_{j=1}^{n}\left(\right.$ trace $\left.W^{i j} H_{i}\right)$

where $\mathrm{W}^{\mathrm{ij}}$ is a $2 \times 2$ symmetric matrices, $\mathrm{H}_{\mathrm{i}}$ denotes the Hessian matrix of I and I represents pixel varying from 1 to $n$. The method illustrates the effective adaptation to restore the blurred edge structures but is also sensitive to impulse noise and speckles which reduce the quality of processed images. Yu et al. [11] used Kernel gradient operator $\nabla(\varnothing \mathrm{I})$ in place of $|\nabla \mathrm{I}|$ in the calculation of diffusion coefficient function (KAD) as shown in Eq. (11).

$d(|\nabla(\emptyset I)|)=\frac{1}{1+\left(\frac{|\nabla(\emptyset I)|}{k}\right)^{2}}$

\begin{tabular}{|c|c|}
\hline Method & Features \\
\hline PMD [3] & $\begin{array}{l}\text { - The blurring and localization } \\
\text { problem of linear diffusion } \\
\text { filtering is removed. } \\
\text { Images close to each other } \\
\text { could produce divergent } \\
\text { solutions with very different } \\
\text { edges. } \\
\text { - Local noise and contrast are } \\
\text { not considered. } \\
\text { - Failure when noise gradient is } \\
\text { greater than edge. } \\
\text { - A large number of iterations } \\
\text { necessary to reach a steady- } \\
\text { state solution } \\
\text { - Longer computation time } \\
\text { along with more blurring } \\
\text { Loss of sharpness at edges. }\end{array}$ \\
\hline CLMCD [5] & $\begin{array}{l}\text { Blurring of fine details and } \\
\text { high gradient edges occur. }\end{array}$ \\
\hline YKD[6] & $\begin{array}{l}\text { Detection of sharp edges and } \\
\text { fine details. } \\
\text { - Sensitive to impulse noise } \\
\text { and speckles. }\end{array}$ \\
\hline CBMD [7] & $\begin{array}{ll}\text { - } & \text { Better Gaussian noise } \\
\text { reduction. } \\
\text { - Not suitable for high gradient } \\
\text { edge preservation. }\end{array}$ \\
\hline GCD [9] & $\begin{array}{l}\text { Time-dependent Schrodinger } \\
\text { wave equation. } \\
\text { When } \theta \text { approaches } \pi / 2 \text { it } \\
\text { becomes very inefficient to } \\
\text { implement complex diffusion. } \\
\text { For small } \theta \text {, there is no } \\
\text { difference from the case of } \\
\text { PM diffusion. }\end{array}$ \\
\hline TDD [10] & $\begin{array}{l}\text { Use of Hessian matrices. } \\
\text { Edges and impulse noisy } \\
\text { pixels are treated equally. }\end{array}$ \\
\hline KAD [11] & $\begin{array}{l}\text { - Selection of optimal choice of } \\
\text { kernel size is an important } \\
\text { issue }\end{array}$ \\
\hline WAD [23] & $\begin{array}{ll} & \text { Enhances Flow like patterns. } \\
\text { - } & \text { Unable to remove noise } \\
\text { effectively. }\end{array}$ \\
\hline DAD [24] & $\begin{array}{ll}\text { Expensive region analysis. } \\
\text { - } & \text { Dictionary of several classes } \\
\text { require rigorous prior } \\
\text { training. }\end{array}$ \\
\hline EAD [25] & $\begin{array}{ll}\text { - } & \text { Reduce computational } \\
\text { complexity. } \\
\text { - } \\
\text { Medical and industrial image } \\
\text { degradation is not considered. }\end{array}$ \\
\hline
\end{tabular}

Table 1: Summary of Features of General Anisotropic Diffusion Methods. 
All the above modified anisotropic diffusion approaches discussed are edge preserving diffusion schemes for images corrupted with additive Gaussian noise.

In the last few decades, numerous anisotropic diffusion techniques are developed for a variety of image applications. Mammogram images [12] are used for early detection and treatment of breast cancer and their visibility can be improved by anisotropic diffusion based contrast enhancement approaches. In opto-acoustic imaging [13], the spatial resolution is bounded to a finite value which need proper reconstruction algorithms to further improve the contrast of such images. Optical coherence tomography [14] detects retinal-related diseases since it can provide high-resolution information of retinal images which helps in the calculation of retinal thickness and lesions. In order to automate the visual examination of low contrast surface imperfection [15] in glass plates, sheet steel, aluminum strips, liquid crystal display panels etc, the enhancement of images is often the basic requirement. Cardiac ultrasound images [2] are used to assess cardiac physiological indicators, coronary heart diseases, diagnosing heart failure covering a wide range of clinical applications. Detection of brain tumor position [16] using MRI and removal of defective pixels from the image is quite challenging. The identification of potential tumors on computer tomography images for the early stage oral cavity cancer detection [17] requires suitable filtering algorithms. In all these applications, there is a wide use of anisotropic diffusion which helps in defect removal along with detail preservation. The biometrics fingerprint identification [18] using anisotropic diffusion is quite popular since fingerprint has a systematic texture with well-ordered local inclination and frequency. There is a wide use of anisotropic diffusion in digital radiography [19] to detect the weld defects in the welding industry. Anisotropic diffusion has also been used in the detection and removal of cracks in digital paintings [20] because the appearance of cracks on paintings deteriorates the quality perceived. Mineralogy, surveillance, agriculture, and astronomical area mostly use hyper spectral images [21] where anisotropic smoothing helps to restore the image features. Remote sensing image [22] helps in detecting vehicles, buildings, road-linked objects and acquisition of transportation data. Classical divergence based Anisotropic approaches include Weickert's edgeenhancing diffusion (WAD) [23], Cho's dictionary-based anisotropic diffusion (DAD) [24] and its extended anisotropic diffusion (EAD) [25] are diffusion techniques available in the literature which are quite effective in all the above image applications.

Table 1 summarizes the above discussed anisotropic diffusion techniques. The major issue with anisotropic diffusion is to deal with speckle noise and poor contrast. The performance of anisotropic diffusion techniques in the field of speckle-noise reduction and low contrast image enhancement are discussed in the following Sections.

\subsection{Speckle noise reduction}

The most dominant type of noise in real world imaging is speckle noise [26][27] which is the form of locally correlated multiplicative noise. The principal causes of this noise are the environmental conditions during image acquisition and the quality of the image sensing elements. This speckle noise has a direct impact on the performance and structural detail of the image under process. A generalized model of speckle noise [26][27] is given as:

$g_{i, j}=I_{i, j} n_{i, j}$

where, $g_{i, j}$ and $I_{i, j}$ are observed speckle noisy image and original image at the pixel position $(i, j)$ resepectively. $n_{i, j}$ is the speckle noise perturbation which is generally assumed to be white Gaussian noise of zero mean and variance $\sigma_{n}{ }^{2}$ at position (i,j). The use of medical imaging is very much affected by such multiplicative speckle noise which degrades its usefulness in diagnosis and modality. But to de-speckle the image without disturbing the essential features like edges and boundaries has always been observed to be very difficult and challenging task. Classical de-speckling filters like Lee [28], Frost [29], Kuan [30] and Gamma [31] inhibit smoothing near edges by using adaptive filters where calculation of coefficient of variation is important. The problem of inaccurate speckle statistical modeling and the issue of poor localization of edges in these window based filter is solved by using non-homogeneous diffusive heat phenomenon. This is state-of-the-art speckle reducing anisotropic diffusion (SRAD) [32] where the diffusion coefficient function has been defined as:

$c(\mathrm{q})=\frac{1}{1+\left[q^{2}(x, y, t)-q_{0}^{2}(t)\right] /\left[q_{0}^{2}(t)\left(1+q_{0}^{2}(t)\right)\right]}$

or

$c(q)=\exp \left\{-\frac{\left[q^{2}(x, y, t)-q_{0}^{2}(t)\right]}{\left[q_{0}^{2}(t)\left(1+q_{0}^{2}(t)\right)\right]}\right\}$

where $\mathrm{q}(\mathrm{x}, \mathrm{y}, \mathrm{t})$ is named as instantaneous coefficient of variation which is dependent on $\nabla \mathrm{I}$ and is determined as:

$q(x, y, t)=\sqrt{\frac{\left(\frac{1}{2}\right)(\nabla I / I)^{2}-\left(\frac{1}{4}\right)\left(\nabla^{2} I / I\right)^{2}}{1+\left(\frac{1}{4}\right)\left(\nabla^{2} I / I\right)^{2}}}$

and $q_{0}(t)$ is speckle scale function. The edge preservation sensitivity of this method was further examined [15] and presented as detail preserving anisotropic diffusion where the orientation of edges were made to stabilize while removing speckle noise as indicated in $\mathrm{Eq}(16)$.

$q(x, t)=\frac{\left|\alpha\|\nabla I\|^{2}-\beta\left(\nabla^{2} I\right)^{2}\right|^{1 / 2}}{\left[I+\gamma \nabla^{2} I\right]}$

The $\mathrm{q}(\mathrm{x}, \mathrm{t})$ denotes the edge stabilizing function with $\alpha, \beta$ and $\gamma$ are the regularization parameters. However, the improper selection of these regularization parameters in diffusion equation suffers from over filtering and blurring of edges. Further, edge-sensitive extensions of SRAD are presented in detail preserving anisotropic diffusion (DPAD) [33] and oriented speckle reducing anisotropic diffusion (OSRAD) [34] where DPAD equation as derived from Kuan filter is given by Eq. (17).

$\bar{u}^{t} I_{i, j}^{t+\Delta t}=I_{i, j}^{t}+\frac{\Delta t}{\left|\overline{\eta_{l, j}}\right|} \operatorname{div}\left[c_{2}\left(C_{i, j}\right) \nabla I_{i, j}^{t}\right]$ 
where $\quad c_{2}\left(C_{i, j, t}\right)=1-k_{k}^{i, j, t} \quad$ and $\quad k_{k}^{i, j}=1-\frac{1+\frac{1}{C_{i, j}^{2}}}{1+\frac{1}{C_{u}^{2}}}$.

Optimized Bayesian non-local means (OBNLM) [35] method came out as an improved de-speckling method where Bayesian formulation has been used which is based on nonlocal mean filtering mechanism. A Type-II Fuzzy anisotropic diffusion algorithm (FuzAD) [36] for OCT images was suggested by Puvanathasan and Bizheva by considering uncertainty in the calculated diffusion coefficient. This proves the best edge preserver in comparison to Type-I fuzzy anisotropic diffusion algorithm, wiener filter and Adaptive Lee filter for fairly short processing time. Probability-driven speckle reducing anisotropic diffusion POSRAD [37] uses tissue-based statistical models and shows good result. However, there is still a significant loss of structural details in the restored image. Wu and Tang [38] suggested an anisotropic method for speckle noise removal by considering two functions named as fidelity and speed function based upon ENI (edge, noise, interior pixels). If $\mathrm{p}=(\mathrm{i}, \mathrm{j})$ is pixel under consideration and $N_{P}^{0}(w)$ are neighbor pixels centered at test pixel $\mathrm{p}$ and window size is $\mathrm{w}$, then for each $q \in$ $N_{P}^{0}(w), \mathrm{d}(\mathrm{p}, \mathrm{q})$ is a difference in intensity of pixelp and $\mathrm{q}$. The controlling speed function and the controlling fidelity function are shown in $\mathrm{Eq}(18)$ and (19) respectively.

$$
\begin{aligned}
& g_{n}\left(E N I_{P}\right)=\frac{1}{2}+\frac{1}{2} \cos \left(\frac{2 \pi E N I_{P}}{N}\right) \\
& \lambda_{n}\left(E N I_{P}\right)=\frac{1}{4}-\frac{1}{4} \cos \left(\frac{\pi E N I_{P}}{N}\right) \\
& E N I_{P}=\sum_{q \in N_{P}^{0}(w)} I_{p}(q) \\
& I_{P}(q)= \begin{cases}1 & \text { whend }(p, q) \leq T \\
0 & \text { whend }(p, q)>T\end{cases}
\end{aligned}
$$

where $N=\left((2 w+1)^{2}-1\right)$ denotes the number of pixels in a squared size window w. The value of $g_{n}$ is minimum at edge, maximum at interior pixels and intermediate at noise. The value of $\lambda_{n}$ is minimum at noise, maximum at interior pixels and intermediate at edges. The revised new selective degenerate diffusion (NSDD) model based on Eq. (18) and (19) is given as:

$$
\begin{aligned}
& \quad \frac{\partial I}{\partial t}=g_{n}\left(E N I_{P}(I, w, T)\right)|\nabla I| \operatorname{div}\left(\frac{\nabla \mathrm{I}}{|\nabla \mathrm{I}|}\right)+ \\
& \lambda_{n}\left(E N I_{P}\left(I_{0}, w, T\right)\left(I_{0}-I\right)\right.
\end{aligned}
$$

The condensed anisotropic diffusion model (CAD) [39] uses diffusion term to preserve and enhance edges where as the second regularization term condenses the diffusion and emphasize thin, linear, and point features stabilization. The method was further extended by Nafiset al. [83] where impulse noise initially removed by median filtering and then de-speckling is done by gray level variance controlled anisotropic diffusion. Febrinni et al. [40] proposed a revised anisotropic diffusion filter named as improved edge enhancing diffusion (IEED) to minimize noise on homogeneous regions while keeping weak edges. Although, IEED is less complex and does not use any mathematical modeling of noise, however, it is useful for

\begin{tabular}{|c|c|c|}
\hline Method & $\begin{array}{l}\text { Optimal } \\
\text { Parameters }\end{array}$ & \multirow{21}{*}{$\begin{array}{l}\boldsymbol{Q}_{\mathbf{1}}=\text { Edge } \\
\text { controlling } \\
\text { quantile, } \\
\boldsymbol{Q}_{\mathbf{2}}=\text { Corner } \\
\text { controlling } \\
\text { quantile, } \\
\Delta_{\boldsymbol{t}}=\text { time step, } \\
\boldsymbol{n}_{\text {iter }}=\text { number } \\
\text { of iterations } \\
\boldsymbol{\sigma}, \boldsymbol{\rho}=\text { smoothing } \\
\text { parameter } \\
\text { (ADMSS), } \\
\boldsymbol{W}=\text { window } \\
\text { size } \\
\boldsymbol{h}=\text { smoothing } \\
\text { parameter } \\
\text { (OBNLM), } \\
\boldsymbol{\alpha}=\text { Patch size } \\
\boldsymbol{M}=\text { area } \\
\text { search similar } \\
\text { patches } \\
\boldsymbol{N}_{\boldsymbol{C}}=\text { tissue class } \\
\boldsymbol{n}_{\boldsymbol{m}}=\text { value of } \\
\text { memory element } \\
\boldsymbol{k}_{\boldsymbol{s}}=\text { number of } \\
\text { super pixel } \\
\lambda, \boldsymbol{\alpha}=\text { positive } \\
\text { balance constant } \\
\boldsymbol{\sigma}, \boldsymbol{\rho}=\text { scaling } \\
\text { parameter of } \\
\text { Gaussian kernel } \\
\boldsymbol{T}=\text { Threshold } \\
\text { (NSDD) } \\
\boldsymbol{\sigma}_{\boldsymbol{s} p e c k l e}= \\
\text { standard } \\
\text { deviation } \\
\text { speckle-noise } \\
\text { model } \\
\boldsymbol{V}_{\text {speckle }}= \\
\text { variance } \\
\text { speckle noise }\end{array}$} \\
\hline Lee [28] & $\begin{array}{l}\sigma=0.01 \\
W=7\end{array}$ & \\
\hline Frost [29] & $W=7$ & \\
\hline Kuan [30] & $\begin{array}{l}\sigma=0.01 \\
W=6\end{array}$ & \\
\hline Gamma [31] & $\begin{array}{l}\text { Number of looks }(L) \\
=4000 \\
W=5\end{array}$ & \\
\hline SRAD [32] & $\begin{array}{l}\Delta_{t}=0.3 \\
n_{\text {iter }}=100\end{array}$ & \\
\hline DPAD [33] & $\begin{array}{l}\Delta_{t}=0.3 \\
n_{\text {iter }}=10 \\
W=5\end{array}$ & \\
\hline OSRAD [34] & $\begin{array}{l}\Delta_{t}=0.3 \\
n_{\text {iter }}=20 \\
\sigma=1\end{array}$ & \\
\hline $\begin{array}{l}\text { OBNLM } \\
{[35]}\end{array}$ & $\begin{array}{c}M=67 \\
\alpha=3 \\
h=0.9\end{array}$ & \\
\hline FuzAD [36] & ----- & \\
\hline $\begin{array}{l}\text { POSRAD } \\
\text { [37] }\end{array}$ & $\begin{array}{l}\Delta_{t}=0.5 \\
n_{\text {iter }}=200 \\
\sigma=1 \\
\sigma_{\text {speckle }}=0.5\end{array}$ & \\
\hline NSDD [38] & $\begin{array}{l}W=2 \\
T=20\end{array}$ & \\
\hline CAD [39] & $\begin{array}{l}n_{\text {iter }}=300 \\
\sigma=0.5 \\
\quad=0.05 \\
\Delta_{t} \\
W=7\end{array}$ & \\
\hline IEED [40] & $\begin{array}{l}\Delta_{t}=0.5 \\
n_{\text {iter }}=200 \\
Q_{1}=0.95 \\
Q_{2}=1\end{array}$ & \\
\hline ADMSS [41] & $\begin{array}{l}\Delta_{t}=0.3, n_{\text {iter }}=19, \\
\sigma, \rho=0.005 \\
n_{m}=3 \\
N_{C}=4\end{array}$ & \\
\hline CDAD [44] & $\begin{array}{l}\Delta_{t}=0.5 \\
n_{\text {iter }}=200 \\
\rho=0.18 \\
\text { Cluster number }=5\end{array}$ & \\
\hline $\begin{array}{l}\text { EPPR- } \\
\text { SRAD [45] }\end{array}$ & $\begin{array}{l}\Delta_{t}=0.02 \\
n_{\text {iter }}=145 \\
\sigma=50 \\
k_{s}=35\end{array}$ & \\
\hline NDEB [46] & $\sigma_{\text {speckle }}=2,3,4$ & \\
\hline IDDND [47] & $\sigma_{\text {speckle }}=2,3,4$ & \\
\hline $\begin{array}{l}\text { GAD-LBM } \\
{[48]}\end{array}$ & $\begin{array}{l}V_{\text {speckle }} \\
=0.03,0.12\end{array}$ & \\
\hline $\begin{array}{l}\text { SGS-SRAD } \\
{[49]}\end{array}$ & $\begin{array}{l}\Delta_{t}=V_{\text {speckle }} \\
=0.03,0.120 .1 \\
n_{\text {iter }}=100\end{array}$ & \\
\hline
\end{tabular}
speckle noise reduction. The problem of edge blurring is mildly resolved in ADMSS [41] by extending the formulation of Cottet and Ayyadi [42] to perform selective diffusion so that diffusion across the fine structures and homogeneous regions are discriminated and in this way
Table 2: Summary of Optimal Parameters of Speckle Reducing Ani-sotropic Diffusion Techniques.

visibility of important structures is enhanced. Fick's law based physical diffusion equation theory is used in doubly degenerate nonlinear diffusion (DDND) model by Zhou et al. [43] to promote de-noising process as given below: 
$\frac{\partial I_{t}}{\partial t}=\operatorname{div}\left(b(\mathrm{I}) \frac{1}{\left(1+|\nabla I|^{2}\right)^{(1-\beta) / 2}} \nabla I\right)$ where $\mathrm{b}(\mathrm{I})$ is the gray level indicator function which is utilized to regulate the diffusion process depending on

\begin{tabular}{|c|c|c|}
\hline Method & Advantages & Disadvantages \\
\hline $\begin{array}{l}\text { Lee [28] } \\
\text { Frost [29] } \\
\text { Kuan [30] } \\
\text { Gamma [31] }\end{array}$ & - Successfully inhibit smoothing near edges. & $\begin{array}{l}\text { - All are sensitive to the size and shape of the filter. } \\
\text { - Do not enhance edges. } \\
\text { - These four Filters are not directional. } \\
\text { - Noisy boundaries of sharp features remain unfiltered. } \\
\text { - High CV in window on edge results in less smoothing } \\
\text { but problem occurs when noisy pixel is inside the } \\
\text { window. }\end{array}$ \\
\hline SRAD [32] & $\begin{array}{l}\text { - } \text { Preserve as well as enhance edges. } \\
\text { - Adaptive Filtering. } \\
\text { - In speckle presence, instantaneous CV plays } \\
\text { a crucial role as an edge detector. }\end{array}$ & $\begin{array}{l}\text { - Accidently eliminates some details in the original } \\
\text { image. } \\
\text { - Over-filtering is there. }\end{array}$ \\
\hline DPAD [33] & $\begin{array}{l}\text { - Large window helps to get better results and } \\
\text { stability. } \\
\text { - Detail preservation and noise removal are } \\
\text { better than SRAD. }\end{array}$ & $\begin{array}{l}\text { - An accurate estimation of statistics is required. } \\
\text { - Sometimes meaningful structural details are lost during } \\
\text { iterations. } \\
\text { - Overfiltering occurs. }\end{array}$ \\
\hline OSRAD [34] & $\begin{array}{l}\text { - Local image geometry is considered. } \\
\text { - Reduce noise. } \\
\text { - Preserve and enhance contours. }\end{array}$ & $\begin{array}{l}\text { - Complex statistical models for speckle distribution are } \\
\text { considered }\end{array}$ \\
\hline OBNLM [35] & $\begin{array}{l}\text { - Very efficient speckle removal while } \\
\text { preserving structure. } \\
\text { - Manual tuning. }\end{array}$ & $\begin{array}{l}\text { - Automatic tuning is required in OBNLM filter. } \\
\text { - Less robust. } \\
\text { - Effect on post-processing tasks such as image } \\
\text { registration or image segmentation needs to explore. }\end{array}$ \\
\hline $\begin{array}{l}\text { Type-II Fuzzy } \\
\text { AD [36] }\end{array}$ & $\begin{array}{l}\text { - Logarithmic transformation is utilized to } \\
\text { transform speckle noise to additive noise. } \\
\text { - Less processing time. } \\
\text { - Significant reduction in speckle pattern. }\end{array}$ & $\begin{array}{l}\text { - Applicable only for OCT images. } \\
\text { - Require log transformation. } \\
\text { - New Fuzzy rules can be added to consider more } \\
\text { features of interest. }\end{array}$ \\
\hline POSRAD [37] & $\begin{array}{l}\text { - The tissue-based statistical model is } \\
\text { considered. }\end{array}$ & - Structural loss of details is still there \\
\hline NSDD [38] & $\begin{array}{l}\text { - Random-valued impulse noise is removed } \\
\text { effectively. } \\
\text { - Selective diffusion and fidelity at edge, } \\
\text { noise, and interior pixels. }\end{array}$ & $\begin{array}{l}\text { - There is no explicit formula or method to determine the } \\
\text { parameters } w \text { and } T \text {. } \\
\text { - Results are checked on standard test images. } \\
\text { - The new controlling function can be extended to any } \\
\text { other PDE denoising model. }\end{array}$ \\
\hline CAD [39] & - Better speckle reduction. & $\begin{array}{l}\text { - Explored for different noise. } \\
\text { - Can be explored for image segmentation, feature } \\
\text { extraction. }\end{array}$ \\
\hline IEED [40] & $\begin{array}{l}\text { - Outperform state of art filters for SAR } \\
\text { image despeckling. } \\
\text { - } \text { Preserve small detail and weak edges. } \\
\text { - Computation time is low }\end{array}$ & - Only for Speckle contaminated images. \\
\hline ADMSS [41] & $\begin{array}{l}\text { - } \quad \text { Preserve and enhance relevant tissue detail. } \\
\text { - No over filtering. }\end{array}$ & $\begin{array}{l}\text { - Application on noise other than speckle can be } \\
\text { checked. } \\
\text { - Can be extended for Low-contrast and color images. }\end{array}$ \\
\hline CDAD [44] & $\begin{array}{l}\text { - } \quad \text { Cluster-based automatic smoothing } \\
\text { - } \quad \text { Stable results are obtained }\end{array}$ & $\begin{array}{l}\text { - Checked against SRAD filter only. } \\
\text { - More clustering techniques can be used to perform } \\
\text { AD. }\end{array}$ \\
\hline $\begin{array}{l}\text { EPPR-SRAD } \\
{[45]}\end{array}$ & $\begin{array}{l}\text { - The over-smoothing effect is reduced. } \\
\text { - Good diagnostic quality results for cardiac } \\
\text { and liver US images. }\end{array}$ & $\begin{array}{l}\text { - Computation time is more. } \\
\text { - Can be explored for different noise and colored } \\
\text { images. }\end{array}$ \\
\hline NDEB [46] & - Applicable for low contrast speckled image. & - Can be explored further for industrial image. \\
\hline IDDND [47] & $\begin{array}{l}\text { - Suitable for color image denoising. } \\
\text { - Low contrast Image Enhancement. }\end{array}$ & $\begin{array}{l}\text { - Can be checked on industrial and remote sensing } \\
\text { image. }\end{array}$ \\
\hline GAD-LBM [48] &  & $\begin{array}{l}\text { - Mathematically further can be explored. } \\
\text { - Application on different noisy and color images can } \\
\text { be extended. }\end{array}$ \\
\hline $\begin{array}{l}\text { SGS-SRAD } \\
{[49]}\end{array}$ & $\begin{array}{l}\text { - Good edge preservation as found by Canny } \\
\text { Edge detector. }\end{array}$ & - Inapplicable for low-contrast images. \\
\hline
\end{tabular}

Table 3: Summary of Features of Speckle Reducing Anisotropic Diffusion Techniques. 
image structures. K-means clustering-based anisotropic diffusion filter (CDAD) [44] uses cluster-based speckle scale function to choose the homogeneous sample region and separate it from noisy region. To control and guide the diffusion process in speckle noise images, Mishra et al. [45] uses probability density function of edge pixel relativity information (EPPR-SRAD) to ensure maximum edge preservation. Zhou et al. [46] further modified DDND [43] and give new de-speckling diffusion model (NDEB):

$\frac{\partial I_{t}}{\partial t}=\operatorname{div}\left(\frac{\nabla I}{1+(|\nabla I| / k)^{\beta(I)}}\right)$

where $\beta(I)=\{1-b(I)\}$ is a region indicator function. Gao et al. [47] observed erroneous pixels appear in homogeneous background of the image obtained by ADMSS [41] and therefore they developed an improved DDND model (IDDND) for multiplicative speckle reduction. $\mathrm{Xu}$ et al. [48] suggested Gabor filter based anisotropic diffusion (GAD-LBM), supporting the advantages of the Lattice Boltzmann method [81] on rapid parallel implementation. GAD-LBM diffusion equation is as follows:

$\frac{\partial I_{t}}{\partial t}=\operatorname{div}\left(\frac{2}{3}\left(\frac{1}{R\left(G_{s d}\right)}-\frac{1}{2}\right) \nabla I\right)$

where $R\left(G_{s d}\right)=\frac{2}{1+3 g\left(G_{s d}\right)}$ is a relaxation factor based on $g\left(G_{s d}\right)=\frac{1}{1+\left(G_{s d} / k\right)^{2}}$ which is edge enhanced Gabor diffusion coefficient function. Goyalet al. proposed an SGS-SRAD filter [49] for de-speckling of US images which is a combination of SavitzkyGolay smoothing filter [82] and SRAD [32]. The optimal parameter settings and the important features of all the discussed anisotropic diffusion techniques for speckle noise removal are summarized in Table 2 and Table 3 respectively.

\subsection{Low contrast image enhancement}

The low gray level inter-region edges in low contrast images require proper enhancement to ease the image understanding. Low Contrast medical, geological, industrial, and photographic images exhibit this problem mostly due to imperfect illumination and settings of image sensor at the time of image capturing and acquisition. The simplest way to enhance the contrast of the image is intensity transformation which is also called as histogram stretching [1].The state-of-the-art anisotropic diffusion technique named as local variance-controlled forwardand-backward (LVCFAB) diffusion method is presented by Wang et al. [50] for image intensification along with noise reduction by combining sharpening and smoothing coefficients in forward and backward direction as given in Eq. (26). The algorithm needs to work upon for adaptive parameter selection and lowering of computational complexity. The diffusion coefficient function of this method is:

$C=C_{\text {forward }}+C_{\text {backward }}$

Chao and Tsai [51] suggested anisotropic diffusion based defect detection in glass images where grayscale variation of defects for background is barely discernible. The diffusion model combines smoothing and sharpening in one single equation whose strength is dependent on two separate diffusion coefficient functions $S_{1}$ and $S_{2}$ as given in Eq. (27) and (28) respectively.

$I_{t+1}=I_{t}+\frac{1}{4} \sum_{i=1}^{4}\left[S_{1}\left(\nabla I_{t}\right)-S_{2}\left(\nabla I_{t}\right)\right] \nabla I_{t}$

$S_{2}(\nabla I)=\alpha \cdot\left[1-S_{1}(\nabla I)\right]$

where $\alpha$ is a weighting parameter that lies between 0 and 1. Chao and Tsai [52] further formulated an edgepreserving smoothing technique for restoring noisy low contrast medical and artwork images where the diffusion coefficient with adaptive threshold $\mathrm{k}$ is used as:

$k=\frac{\sigma_{N}^{2}}{k_{0}}$

Here, $\sigma_{N}^{2}$ is normalized variance and $k_{0}$ is a constant. Chao and Tsai [53] give a diffusion coefficient function based on logarithmic function to control diffusion by varying the parameters $\alpha, \beta$ and $\gamma$ as given below:

$d(\nabla I)=\alpha \cdot \ln (\nabla f+\beta)+\gamma$

This method proves beneficial for brightness enhancement films in industrial applications. Nafis et al. [54] used histogram statistics to find adaptive threshold parameter in diffusion equation as given in Eq. (31).

$k^{\prime}=\frac{1}{1+e^{-\left(a_{0} \sigma_{g}^{2}-\sigma_{w}^{2}\right)}}$

where $\sigma_{g}^{2}$ and $\sigma_{w}^{2}$ are global and local gray level variance with $\mathrm{w}$ as a local window size and $a_{0}$ is a constant. This method is quite effective in generalized low contrast images. Robust coherence enhancing diffusion (RCED) and robust anisotropic diffusion (RAD) filters developed by Ham et al. [55] by combining anisotropic diffusion with adaptive smoothing based on asymmetric diffusion flow instead of symmetric as in the case of anisotropic diffusion. This method was quite effective in enhancing the tiny and coherent structures. However, it is not suitable for noisy images. Wang et al. [21] suggested gradient and laplacian based hyperspectral anisotropic diffusion (GLHAD) algorithm by introducing the Laplacian function into classical PMD [3] for HSI improvement. The operational utility of GLHAD method needs to be improved further by proper parameter selection and optimization. In Weickert's diffusion method [56], seismic image filtering result is much better than PMD [3] and also effective in single dimensional audio signals but still some undesirable oscillations and artifacts are observed in the output. Zhou et al. [57] proposed adaptive parameter time fractional-order anisotropic diffusion filtering which attenuates noise with minimal impact on desirable seismic information in comparison to PMD [3] and Weickert [56] and thus helps in seismic data processing and interpretation. Zanget al. [22] applied a similar idea of image enhancement for road network extraction. Ben Gharsallah [58] suggested geometric smoothing and sharpening anisotropic diffusion (GSSAD) in welding inspection of radiographic images for detection of defects which may affect the well-functioning of many electro-mechanical systems. Here, the diffusion function is calculated in $\mathrm{x}$ and $\mathrm{y}$ direction. The local geometric variables are computed first which are $D_{1 x}, D_{1 y}, P_{1 x}, P_{1 y}$ and a are weighting factor. The two weighting smoothing functions $\left(g_{1 x}\left(D_{x}, P_{x}\right)\right.$ and $\left.g_{1 y}\left(D_{y}, P_{y}\right)\right)$ and the two sharpening functions $\left(v_{1 x}\right.$ and $\left.v_{1 y}\right)$ are shown in Eq (32), (33), (34) and (35) respectively. 


$$
\begin{aligned}
& g_{1 x}\left(D_{x}, P_{x}\right)=\frac{2}{1+\exp a\left|\frac{D_{1 x}}{P_{1 x}}\right|} \\
& g_{1 y}\left(D_{y}, P_{y}\right)=\frac{2}{1+\exp a\left|\frac{D_{1 y}}{P_{1 y}}\right|} \\
& v_{1 x}=\alpha_{1}\left(1-g_{1 x}\right) \\
& v_{1 y}=\alpha_{1}\left(1-g_{1 y}\right)
\end{aligned}
$$

These separate diffusion coefficients for smoothing and sharpening improve the control of the diffusion process in radiography. Chen et al. [59] gives selfadaptive tangential and normal diffusion coefficients. Diffusion coefficient $\alpha$ based on local variance $\sigma^{2}$ and threshold $\mathrm{T}$ is given as:

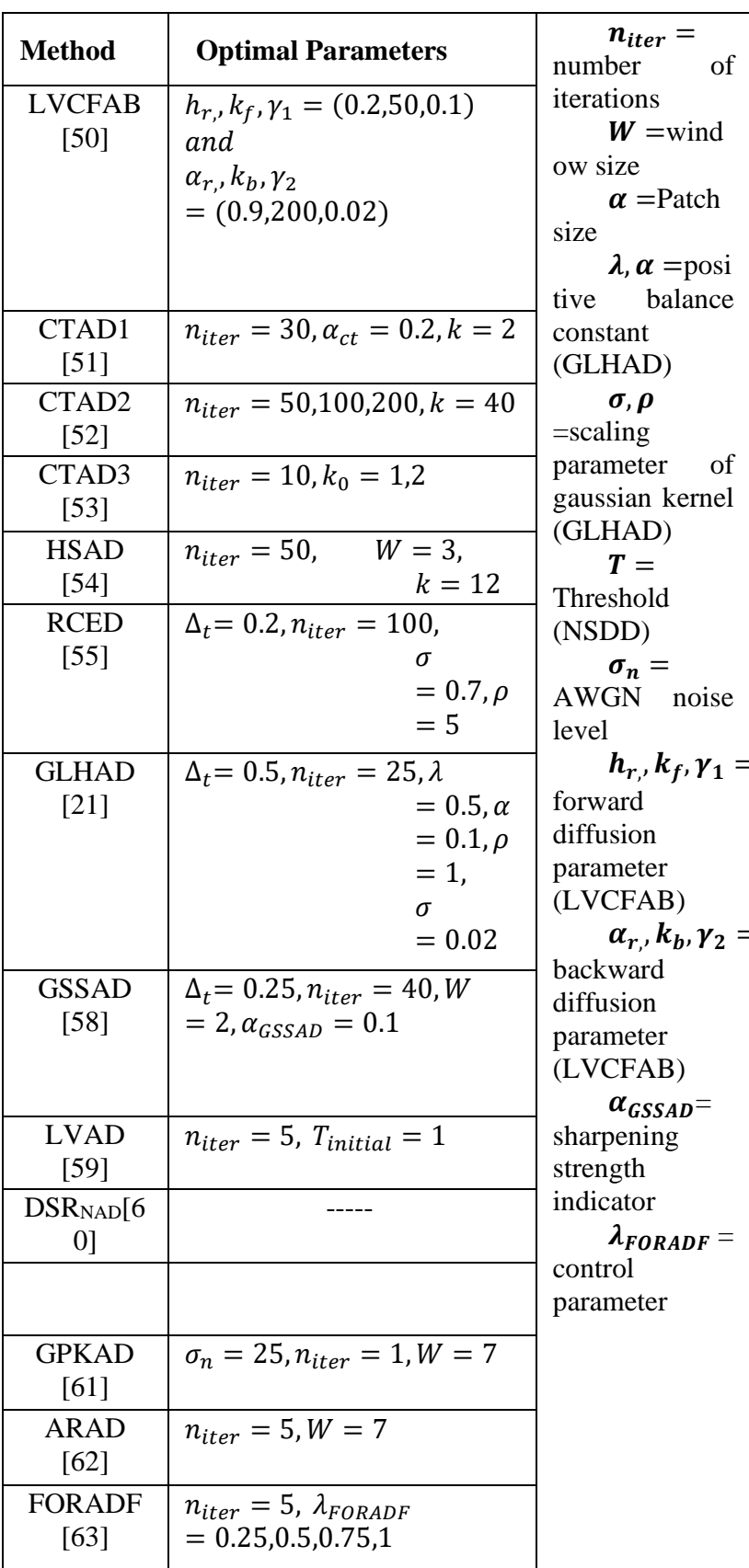

Table 4: Summary of Optimal Parameters of Anisotropic Diffusion Techniques for Low-Contrast Image Enhancement. $\alpha\left(\sigma^{2}\right)=\left\{\begin{array}{c}\cos \left(\frac{\pi}{T} \sigma^{2}\right) \text { when } \sigma^{2} \leq T \\ \cos \left(\frac{\pi}{2(255-T)}\left(\sigma^{2}-3 T+510\right)\right) \text { when }^{2}>T\end{array}\right.$

Liu and Zhang [60] combined non-linear diffusion with dynamic stochastic resonance DSR $_{\mathrm{NAD}}$ to enhance dark and low contrast images but they found poor texture preservation however edges are enhanced successfully. Cho and Kang [61] used geodesic path kernel-based diffusion (GPKAD) for adaptive smoothing with contrast stretching. Chen et al. [62] detect defects in anti-reflective glass images and replacesk in Eq. (2) by $1 / \mathrm{Z}$ as a variable edge threshold where $\mathrm{z}$ is defined as:

$z=\frac{f(x, y)-\mu}{\sigma}$

where $\mu$ and $\sigma$ are local mean and standard deviation. The computation in this method (ARAD) [62] is fast but regardless of whether the edge strength of a defective object is low or high, the edge gets enhanced and therefore the applications of ARAD in medical imaging require more modifications in diffusion equation. NDEB [46] and IDDND [47] discussed in section 3.1 can be included for low contrast speckle contaminated images too. Nair et al. [63] proposed first-order anisotropic diffusion (FORADF) model which is given as:

$I_{t+1}=I_{t}+\alpha C .\left\{\operatorname{Med}\left[\left(\nabla_{N} I_{t}\right),\left(\nabla_{S} I_{t}\right),\left(\nabla_{E} I_{t}\right),\left(\nabla_{W} I_{t}\right)\right]\right\}$

where $0<\alpha<1$ and N,S,E,W are four neighbors of center pixel. The diffusion coefficient $\mathrm{C}$ in this model is defined as:

$C=\exp .\left(-\left(\operatorname{Med}\left[\nabla_{t} I\right] / k\right)\right)$

Where Med denote the median of the gradient magnitudes calculated in the four main directions and $\mathrm{k}$ is threshold constant. Chen et al. [64] proposed an optimal anisotropic diffusion by combining the concept of artificial neural network with local gradient which effectively distinguishes image structures and textures.

A summary of optimal parameters and features of different anisotropic diffusion methods discussed for low contrast images are shown in Table 4 and 5 respectively.

\section{Performance analysis and assessment}

In order to validate the performance of image enhancement and d-noising methods, various image quality assessment (IQA) techniques [65][66][67] are available in the literature. The IQA parameters used for anisotropic diffusion based enhancement and de-noising methods are presented in this Section. The de-speckling performance is mostly measured by Equivalent Number of Looks (ENL) [40] given as:

$E N L=\left(\frac{4}{\pi}-1\right) \frac{\mu_{I}^{2}}{\sigma_{\tilde{I}}^{2}}$

where $\mu_{\tilde{I}}^{2}$ and $\sigma_{\tilde{I}}^{2}$ are mean and variance of de-speckled image I. Contrast Measure (CM) [44] is used to check the performance of contrast improvement in de-speckling algorithms which can be defined as:

$\mathrm{CM}=\frac{1}{\mathrm{n}} \sum_{\mathrm{m}}|\mathrm{c}(\mathrm{i})| \cdot \log (1+|\mathrm{c}(\mathrm{i})|)$ 


\begin{tabular}{|c|c|c|}
\hline Method & Advantages & Disadvantaages \\
\hline $\begin{array}{l}\text { LVCFAB } \\
{[50]}\end{array}$ & $\begin{array}{l}\text { - Do sharpening as well as smoothing. } \\
\text { - Good choice for industrial image de- } \\
\text { noising. }\end{array}$ & $\begin{array}{l}\text { - Adaptive parameter selection is required. } \\
\text { - Computational complexity needs to be } \\
\text { reduced. } \\
\text { - Extended for color images. }\end{array}$ \\
\hline $\begin{array}{l}\text { CTAD1 } \\
{[51]}\end{array}$ & $\begin{array}{l}\text { - Detects defects in low contrast } \\
\text { surface images. } \\
\text { - Sharpening is introduced along with } \\
\text { smoothing. }\end{array}$ & - Extended for image restoration. \\
\hline $\begin{array}{l}\text { CTAD2 } \\
{[52]}\end{array}$ & $\begin{array}{l}\text { - Maintains fine details including local } \\
\text { characteristics. }\end{array}$ & - Fail on high-level noise and impulse noise. \\
\hline CTAD3 & $\begin{array}{l}\text { - Automatic parameter selection by } \\
\text { Particle Swam Optimization. } \\
\text { - Less computation time. } \\
\text { - Effective and efficient defect } \\
\text { detection. }\end{array}$ & - Applicable only for non-textured surface. \\
\hline HSAD [54] & $\begin{array}{l}\text { - Low gray level inter-region edges } \\
\text { are detected. }\end{array}$ & $\begin{array}{l}\text { - Checked for speckle and high dense noise } \\
\text { condition. }\end{array}$ \\
\hline RCED [55] & $\begin{array}{l}\text { - } \text { Adaptive Smoothing. } \\
\text { - Robust. }\end{array}$ & $\begin{array}{l}\text { - Applied on Medical images. } \\
\text { - Quality measure is not employed for } \\
\text { validation. }\end{array}$ \\
\hline $\begin{array}{l}\text { GLAHD } \\
{[21]}\end{array}$ & - Good edge preservation for HSI. & $\begin{array}{l}\text { - } \text { Parameter selection is an issue. } \\
\text { - Computation time needs to be reduced. }\end{array}$ \\
\hline GSSAD & $\begin{array}{l}\text { - Defect detection and segmentation } \\
\text { are good. }\end{array}$ & - Can be explored for Medical imaging also. \\
\hline LVAD [59] & $\begin{array}{l}\text { Local variance calculation facilitates } \\
\text { the difference between noise and } \\
\text { details. }\end{array}$ & $\begin{array}{l}\text { - Compared with linear filtering only. } \\
\text { - Parameter setting needs regulation. }\end{array}$ \\
\hline $\operatorname{DSR}_{\mathrm{NAD}}[60]$ & - Good perceptual quality & - No texture preservation. \\
\hline $\begin{array}{l}\text { GPKAD } \\
{[61]}\end{array}$ & - Better detail preservation. & $\begin{array}{l}\text { - Anisotropic diffusion based CNN denoiser } \\
\text { model can be developed. } \\
\text { - Performance is checked for AWGN only. }\end{array}$ \\
\hline ARAD [62] & 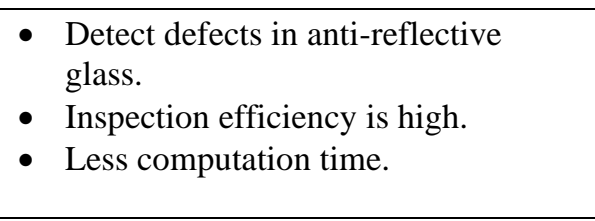 & $\begin{array}{l}\text { - Applied on other surface images. } \\
\text { - Equal enhancement of low and high contrast } \\
\text { defects. } \\
\text { - Problem in Defect segmentation and } \\
\text { classification. }\end{array}$ \\
\hline $\begin{array}{l}\text { FORADF } \\
{[63]}\end{array}$ & $\begin{array}{l}\text { - Low arithmetic complexity } \\
\text { - Robust and power-efficient. }\end{array}$ & $\begin{array}{l}\text { - Can be checked for industrial and medical } \\
\text { images. }\end{array}$ \\
\hline
\end{tabular}

Table 5: Summary of Features of Anisotropic Diffusion Techniques for Low-Contrast Image Enhancement.

where $\mathrm{n}$ and $\mathrm{m}$ are the number of pixels and edge points respectively. c(i) is local contrast at $i^{\text {th }}$ pixel which is calculated as $\mathrm{c}(\mathrm{i})=\sum_{\mathrm{p}}\left(\mathrm{i}-i^{\prime}\right)$ where $\mathrm{p}$ denotes neighbor pixels around i. Puvanathasan and Bizheva [36] utilized Contrast-to-Noise Ratio (CNR) which is the difference between particular image features relative to background which is as given in Eq. (42).

$\mathrm{CNR}=\frac{1}{\mathrm{R}}\left(\sum_{\mathrm{r}=1}^{\mathrm{R}} \frac{\left(\mu_{\mathrm{r}}-\mu_{\mathrm{b}}\right)}{\sqrt{\sigma_{\mathrm{r}}^{2}+\sigma_{\mathrm{b}}^{2}}}\right)$

where $\mu_{\mathrm{b}}$ and $\sigma_{\mathrm{b}}^{2}$ are mean and variance of background noise. $\mu_{\mathrm{r}}$ and $\sigma_{\mathrm{r}}^{2}$ are mean and variance of $r^{\text {th }}$ region of interest. Pratt and Wiley [68] uses Pratt's Figure of Merit
(FOM) to calculate edge preservation in de-noised images. FOM range is between 0 and 1 where 1 represents the best edge detection. FOM score decreases with an increase in the noise level.

$F O M=\frac{1}{\max \{\hat{n}, n\}} \sum_{i=1}^{\widehat{N}} \frac{1}{1+d_{i}^{2} \gamma}$

where $\hat{n}$ and $n$ are detected and reference pixel, $d_{i}$ is Euclidean distance between $\hat{l}_{\text {th }}$ edge pixel and its nearest neighbor and $\gamma=0.9$. Narvekar and Karam [69] used Cumulative Probability Blur Detection (CPBD) where reference image is unknown. Perceptual fog density is calculated by Fog-Aware Density Evaluator (FADE) [70] where low FADE is desired. Wang and Bovik [71] presented a full-reference image quality metric which is a Universal Image Quality Index (UQI). Wang et al. [72] 
and Brooks et al. [73] presented Mean Structural Similarity Index Measurement (MSSIM) by combining luminance similarity, contrast similarity, and structure similarity between reference image $\mathrm{x}$ and the distorted image $\mathrm{y}$ as shown in Eq.(44).

MSSIM $=\left[I(x, y)^{\alpha} \cdot C(x, y)^{\beta} \cdot S(x, y)^{\gamma}\right]$

where I, $\mathrm{C}$ and $\mathrm{S}$ are luminance, contrast and structure measurement with $\alpha, \beta$ and $\gamma$ are constants. Wang and Bovik [74] presented a simple and very popular Mean Square Error (MSE) and Peak Signal to Noise Ratio (PSNR) error sensitivity metric which can be calculated using Eq (45) and (46) respectively.

$M S E=\frac{1}{M N} \sum_{\mathrm{i}=1, \mathrm{j}=1}^{\mathrm{i}=\mathrm{N} . \mathrm{N}}\left(\mathrm{f}_{\mathrm{o}}-\mathrm{f}_{\mathrm{d}}\right)^{2}$

$P S N R=20 \log _{10}\left(\frac{\operatorname{Max}_{f}}{\sqrt{M S E}}\right)$

Where $\mathrm{f}_{\mathrm{o}}$ and $\mathrm{f}_{\mathrm{d}}$ are original and de-noised image respectively. $\operatorname{Max}_{f}$ is the maximum possible pixel intensity of $\mathrm{f}_{\mathrm{o}}$. Chao and Tsai [51] used 3-sigma statistical control based threshold $\left(\mu_{d}+S \sigma_{d}\right)$ where $\mu_{d}$ and $\sigma_{d}$ are mean and standard deviation of diffused image. $S$ denotes the control constant set to 3. Ding et al. [75] introduced Directional Anisotropic Structure Measurement (DASM) by thorough study of structures and textures, to represent visually important dominant structures which is defined as:

$\operatorname{DASM}(p)=G(p) \cdot A(p) \cdot D(p)$

where $p$ is the pixel under test. $G(p), A(p)$ and $D(p)$ are gradient of $\mathrm{p}$, intensity change in local structure and structural direction respectively. Liu and Zhang [60] used Relative Contrast Enhancement Factor (RCEF) defined as follows:

$R C E F=\frac{\sigma_{\tilde{u}}^{2}}{\mu_{\tilde{u}}} \cdot \frac{\mu_{f}}{\sigma_{f}^{2}}$

where $\sigma^{2}$ and $\mu$ denotes mean and variance of the observed image $\tilde{\mu}$ and input low contrast image $\mathrm{f}$ respectively. Another Distribution Separation Measure (DSM) determines the enhancement of the target area relative to the background is shown in Eq. (49).

$D S M=\left(\left|\mu_{\widetilde{u}}^{T}-\mu_{\widetilde{u}}^{B}\right|\right)-\left(\left|\mu_{f}^{T}-\mu_{f}^{B}\right|\right)$

where $\mathrm{T}$ and $\mathrm{B}$ are the target and background area of the observed image respectively. LIVE dataset [76] and TID dataset [77] are popular databases that are used for taking standard images for verification of different algorithms. Table 6 and Table 7 demonstrate the comparative analysis of different anisotropic diffusion methods in terms of all these IQA parameters for speckle noise reduction and contrast improvement respectively.

\section{Observations and discussions}

Anisotropic diffusion based image smoothing with edge preservation is a growing field of research since it is a key factor for successful image enhancement, de-noising, segmentation, classification and recognition. The goal of this study is to review existing anisotropic diffusion filtering techniques and to address several challenging problems for image enhancement and de-noising. A lot of work in this area have been done in past to improve the visual quality of images. However, there are still some new directions of research in anisotropic diffusion for achieving better image enhancement and de-noising. Some properties of diffusion coefficient function are not yet fully explored and thus have sufficient scope to develop more effective diffusion filters.

An important research issue in anisotropic diffusion approaches is the selection of optimal threshold parameter in diffusion coefficient function which can be further explored in order to deal with the higher noise content and better edge preservation. In most of the related literature [48] [49] [52] [53] [54], the edge threshold has been considered as a function of image contents which is a good idea for more robust results. However, as the noise level increases or in the edge abundant areas, this scheme failed to protect maximum edges. Therefore, in this regard, a proper image content analysis is required for formulating the edge threshold function as well as the diffusion coefficient function.

The parameter adjustments in many of the anisotropic diffusion methods [9] [10] [13] [14] [15] [21] [22] [34] [36] [83] required lot of experimentations and it also expands the computational burden. This reveals the user to worry about the parameter settings for all the images in the entire experiment. However, it will be a good idea to explore the possibility of developing some optimization technique to optimize these parameters which also helps in reducing the overall computational burdens. Another important issue where the computational complexity plays an important role is researching the choice of better window size or to develop an adaptive window mechanism for neighborhood operation in images which can perhaps provide efficient enhancement and de-noising with reduced time and space requirements.

There are many emerging approximation and optimization tools available in the literature where most of them are based on soft computing techniques like fuzzy logic and artificial neural network (ANN), singular value decomposition (SVD), principal component analysis (PCA), swarm optimization (SA) and genetic algorithm (GA) etc. These all are very useful in various fields of mathematical science and engineering where parameter settings are required. However, there are very few techniques available in the literature [35] [36] [55] [80] [84] [85] [86] where these soft computing tools have been utilized in anisotropic diffusion filters. This motivates to explore the properties of the soft computing based techniques to develop more approximated and optimized anisotropic diffusion model for specific image applications. The anisotropic diffusion approaches could be further studied for various scientific and industrial image applications such as image segmentation, object recognition and morphological image operations etc.

\section{Conclusion}

A comprehensive survey on image enhancement and denoising by partial differential equation based anisotropic diffusion method is presented in this paper. The physical background of anisotropic diffusion and its application in image enhancement and denoising is discussed. The advancements of anisotropic diffusion in speckle noise reduction and contrast improvement have been explained. 


\begin{tabular}{|c|c|c|}
\hline Method & IQA & Image Type \\
\hline SRAD [32] & FOM & US and SAR Images \\
\hline DPAD [33] & MSSIM & US Image \\
\hline OSRAD [34] & FOM & 2D and 3D Synthetic image \\
\hline OBNLM [35] & ENL & 2D and 3D Synthetic image, Real US image \\
\hline $\begin{array}{l}\text { Type-II Fuzzy AD } \\
{[36]}\end{array}$ & PSNR, ENL, CNR & OCT fingertip and retina Images \\
\hline POSRAD [37] & FOM & Cardiac US image \\
\hline NSDD [38] & PSNR & Standard Test Images (Lena \& Pepper) \\
\hline CAD [39] & FOM, MSE, SNR, MSSIM & Real US and Simulated image \\
\hline IEED [40] & ENL Index & SAR Image \\
\hline ADMSS [41] & ENL, PSNR, SSIM & US image \\
\hline CDAD [44] & $\mathrm{CM}$ & US image \\
\hline EPPR-SRAD [45] & SSIM, MSE, FOM & Synthetic and Real US Cardiac \& Liver images \\
\hline NDEB [46] & MSE, PSNR, MSSIM & $\begin{array}{c}\text { Test Image, Real and synthetic US Image (Low } \\
\text { contrast) }\end{array}$ \\
\hline IDDND [47] & MSE, PSNR,SSIM & $\begin{array}{c}\text { Real ultrasound and RGB color images (Low } \\
\text { contrast) }\end{array}$ \\
\hline GAD-LBM [48] & SSIM,PSNR,FOM & Synthetic and clinical images \\
\hline SGS-SRAD [49] & SSIM, MSE, PSNR & Real and synthetic US images \\
\hline
\end{tabular}

Table 6: Comparative Analysis of Speckle reducing Anisotropic Diffusion Techniques.

\begin{tabular}{|c|c|c|c|}
\hline Method & IQA & Image Type & Noise \\
\hline LVCFAB [50] & PSNR, CNR, SSIM & $\begin{array}{c}\text { Low contrast Standard test image, } \\
\text { MR image }\end{array}$ & Gaussian noise, Blur \\
\hline CTAD2 [52] & PSNR & Artwork, Medical and Test Image & Gaussian noise, Blur \\
\hline HSAD [54] & $\mathrm{CM}$ & Test image & Gaussian noise and blur \\
\hline GLAHD [21] & PSNR, MSSIM & Synthetic and Real HSI & $\begin{array}{c}\text { Gaussian noise, Blur, Impulse } \\
\text { noise }\end{array}$ \\
\hline GSSAD [58] & MSE, PSNR & $\begin{array}{c}\text { Synthetic Image, Real Weld } \\
\text { Radiography Images }\end{array}$ & $\begin{array}{c}\text { Gaussian noise, Blur, Impulse } \\
\text { noise }\end{array}$ \\
\hline LVAD [59] & MSE, PSNR & Test Images & Gaussian noise and blur \\
\hline $\mathrm{DSR}_{\mathrm{NAD}}[60]$ & $\begin{array}{l}\text { RCEF, PQM , } \\
\text { DSM }\end{array}$ & $\begin{array}{c}\text { Cell Phone Image (dark), MR } \\
\text { Image, Video surveillance Image }\end{array}$ & All noise type \\
\hline GPKAD [61] & $\begin{array}{l}\text { MSE, PSNR, } \\
\text { MSSIM }\end{array}$ & Test Images & Gaussian Noise \\
\hline ARAD [62] & $\mathrm{CM}$ & Low contrast Surface Image & Blur \\
\hline FORADF [63] & MSE, PSNR, SSIM & Standard test Images & $\begin{array}{l}\text { Gaussian noise, Impulse } \\
\text { noise, mixed-noise }\end{array}$ \\
\hline
\end{tabular}

Table 7: Comparative Analysis of Anisotropic Diffusion Techniques for Low-Contrast Image Enhancement.

The important critics in state-of-the-art methods are identified. The possible future scopes and research issues in this field have been taken out for the discussion.

Anisotropic diffusion filters are indeed the best option for speckle contaminated and low contrast images. However, a strong and exhaustive exploration for defining optimal diffusion coefficient function is required for some specific type of images. Medical images require such anisotropic diffusion techniques by proper formulation of the diffusion equation since the presence of even small artifacts leads to false diagnosis. The quality of low contrast images for industrial applications can be improved by examining the image feature analysis in order to formulate the diffusion coefficient function. Further to benchmark various anisotropic diffusion technique, more advanced image quality metric can be explored depending upon the type and availability of image under processing. 


\section{References}

[1] Gonzalez, R. C. and Woods, R. E., Digital Image Processing (4th Edition), Pearson Education India, 2018.

[2] Goyal, B., Agrawal, S., \&Sohi, B. S., Noise Issues Prevailing in Various Types of Medical Images, Biomedical \& Pharmacology Journal, 11(3):1227, 2018.

[3] Perona, P., \& Malik, J., Scale-space and edge detection using anisotropic diffusion, IEEE Transactions on Pattern Analysis and Machine Intelligence, 12(7), 629-639, 1990.

[4] Bird. R.B., Transport phenomena, Applied Mechanics Reviews, 55:R1, 2002.

[5] Catté, F., Lions, P. L., Morel, J. M., \&Coll, T., Image selective smoothing and edge detection by nonlinear diffusion, SIAM Journal on Numerical Analysis, 29(1):182-193, 1992.

[6] You, Y. L., \&Kaveh, M., Fourth-order partial differential equations for noise removal, IEEE Transactions on Image Processing, 9(10): 1723-1730, 2000.

[7] Chen, Y., Barcelos, C. A. Z., \&Mair, B. A., Smoothing and edge detection by time-varying coupled nonlinear diffusion equations, Computer Vision and Image Understanding, 82(2): 85-100, 2001.

[8] Weeratunga, S. K., \& Kamath, C., PDE-based nonlinear diffusion techniques for denoising scientific and industrial images: an empirical stud, Image Processing: Algorithms and Systems, Vol. 4667: 279-290, 2002.

[9] Gilboa, G., Sochen, N., \&Zeevi, Y. Y., Image enhancement and denoising by complex diffusion processes, IEEE Transactions on Pattern Analysis and Machine Intelligence, 26(8):1020-1036, 2004.

[10] Tschumperle, D., \&Deriche, R., Vector-valued image regularization with PDEs: A common framework for different applications, IEEE Transactions on Pattern Analysis and Machine Intelligence, 27(4): 506-517, 2005.

[11] Yu, J., Wang, Y., \&Shen, Y., Noise reduction and edge detection via kernel anisotropic diffusion, Pattern Recognition Letters, 29(10): 1496-1503, 2008.

[12] Gupta, B., Tiwari, M., \&Lamba, S. S., Visibility improvement and mass segmentation of mammogram images using quantile separated histogram equalization with local contrast enhancement,CAAI Transactions on Intelligence Technology, 4(2): 7379, 2019.

[13] Hailong, H., Mandal, S., Buehler, A., Deán-Ben, X. L., Razansky, D., \&Ntziachristos, V., Improving opto-acoustic image quality via geometric pixel super-resolution approach, IEEE Transactions on Medical Imaging, 35(3): 812-818, 2015.

[14] Fernandez, D. C., Delineating fluid-filled region boundaries in optical coherence tomography images of the retina, IEEE Transactions on Medical Imaging, 24(8): 929-945, 2005.

[15] Li, W. C., \& Tsai, D. M., Defect inspection in lowcontrast LCD images using Hough transform-based nonstationary line detection, IEEE Transactions on Industrial Informatics, 7(1): 136-147, 2009.

[16] Rashid, M. H. O., Mamun, M. A., Hossain, M. A., \& Uddin, M. P., Brain Tumor Detection Using Anisotropic Filtering, SVM Classifier and Morphological Operation from MR Images, In International Conference on Computer, Communication, Chemical, Material and Electronic Engineering (IC4ME2) (pp. 1-4), 2018

[17] Hu, Z., Alsadoon, A., Manoranjan, P., Prasad, P. W. C., Ali, S., \&Elchouemic, A., Early-stage oral cavity cancer detection: Anisotropic preprocessing and fuzzy C-means segmentation, In IEEE 8th Annual Computing and Communication Workshop and Conference (CCWC), (pp. 714-719), 2018.

[18] Khan, T. M., Bailey, D. G., Khan, M. A., \& Kong, Y., Efficient hardware implementation for fingerprint image enhancement using anisotropic Gaussian filter, IEEE Transactions on Image processing, 26(5): 2116-2126, 2017.

[19] Abd Halim, S., Ibrahim, A., \&Manurung, Y. H., PDE-based model for weld defect detection on digital radiographic image, International Journal of Signal Processing Systems, 1(2): 146-151, 2013.

[20] Giakoumis, I., Nikolaidis, N., \& Pitas, I., Digital image processing techniques for the detection and removal of cracks in digitized paintings, IEEE Transactions on Image Processing, 15(1):178-188, 2005.

[21] Wang, Y., Wu, K., Yang, Y., \& Chen, T., Gradient and Laplacian-Based Hyperspectral Anisotropic Diffusion, IEEE Journal of Selected Topics in Applied Earth Observations and Remote Sensing, 8(6): 3235-3249, 2015.

[22] Zang, Y., Wang, C., Yu, Y., Luo, L., Yang, K., \& Li, J., Joint enhancing filtering for road network extraction, IEEE Transactions on Geoscience and Remote Sensing, 55(3): 1511-1525, 2016.

[23] Weickert, J., Coherence-enhancing diffusion filtering, International Journal of Computer Vision, 31(23):111-127, 1999.

[24] Cho, S. I., Kang, S. J., Kim, H. S., \& Kim, Y. H., Dictionary-based anisotropic diffusion for noise reduction, Pattern Recognition Letters, 46:36-45, 2014.

[25] Cho, S. I., Kang, S. J., Lee, S., \& Kim, Y. H., Extended-dimensional anisotropic diffusion using diffusion paths on inter-color planes for noise reduction, Digital Signal Processing, 48: 27-39, 2016.

[26] Tur, M., Chin, K. C., \& Goodman, J. W., When is speckle noise multiplicative? Applied optics, 21(7): 1157-1159, 1982.

[27] Wagner, R. F., Statistics of speckle in ultrasound Bscans, IEEE Transactions on Sonics \&Ultrasoics, 30(3):156-163, 1983

[28] Lee, J. S. (1980). Digital image enhancement and noise filtering by use of local statistics, IEEE Tansactions on Pattern Analysis and Machine Intelligence, (2):165-168, 1980.

[29] Frost, V. S., Stiles, J. A., Shanmugan, K. S., \&Holtzman, J. C., A model for radar images and its application to adaptive digital filtering of multiplica- 
tive noise, IEEE Tansactions on Pattern Analysis and Machine Intelligence, (2):157-166, 1982.

[30] Kuan, D. T., Sawchuk, A. A., Strand, T. C., \&Chavel, P., Adaptive noise smoothing filter for images with signal-dependent noise, IEEE Tansactions on Pattern Analysis and Machine Intelligence, (2):165-177, 1985.

[31] Lopes, A.,Touzi, R., \&Nezry, E., Adaptive speckle filters and scene heterogeneity, IEEE transactions on Geoscience and Remote Sensing, 28(6): 992-1000, 1990.

[32] Yu, Y., \& Acton, S. T., Speckle reducing anisotropic diffusion, IEEE Transactions on Image Processing, 11(11): 1260-1270, 2002.

[33] Aja Fernández, S. \&Alberola-López, C., On the estimation of the coefficient of variation for anisotropic diffusion speckle filtering, IEEE Transactions on Image Processing, 15(9): 2694-2701, 2006.

[34] Krissian, K., Westin, C. F., Kikinis, R., \&Vosburgh, K. G., Oriented speckle reducing anisotropic diffusion, IEEE Transactions on Image Processing, 16(5): 1412-1424, 2007.

[35] Coupé, P., Hellier, P., Kervrann, C., \&Barillot, C., Nonlocal means-based speckle filtering for ultrasound images, IEEE Transactions on Image Processing, 18(10): 2221-2229, 2009.

[36] Puvanathasan, P., \&Bizheva, K., Interval type-II fuzzy anisotropic diffusion algorithm for speckle noise reduction in optical coherence tomography images, Optics Express, 17(2): 733-746, 2009.

[37] Vegas-Sanchez-Ferrero, G., Aja-Fernandez, S., Martín-Fernández, M., Frangi, A. F., \&Palencia, C., Probabilistic-driven oriented speckle reducing anisotropic diffusion with application to cardiac ultrasonic image, International Conference on Medical Image Computing and Computer-Assisted Intervention (pp. 518-525), Springer, Berlin, 2010.

[38] Wu, J., \& Tang, C., PDE-based random-valued impulse noise removal based on new class of controlling functions, IEEE Transactions on Image Processing, 20(9): 2428-2438, 2011.

[39] Shanmugam, K.,\&Wahidabanu, R. S. D., Condensed anisotropic diffusion for speckle reducton and enhancement in ultrasonography, EURASIP Journal on Image and Video Processing, 2012.

[40] Fabbrini, L., Greco, M., Messina, M., \&Pinelli, G., Improved edge enhancing diffusion filter for speckle-corrupted images, IEEE Geoscience and Remote Sensing Letters, 11(1): 99-103, 2014.

[41] Ramos-Llordén, G., Vegas-Sánchez-Ferrero, G., Martin-Fernandez, M., Alberola-López, C., \& AjaFernández, S., Anisotropic diffusion filter with memory based on speckle statistics for ultrasound images, IEEE Transactions on Image Processing, 24(1):345-358, 2014.

[42] Cottet, G. H., \&Ayyadi, M. E., A Volterra type model for image processing, IEEE Transactions on Image Processing, 7(3):292-303, 1998.

[43] Zhou, Z., Guo, Z., Dong, G., Sun, J., Zhang, D., \& Wu, B., A doubly degenerate diffusion model based on the gray level indicator for multiplicative noise removal, IEEE Transactions on Image Processing, 24(1): 249-260, 2015.

[44] Hu, Z., \& Tang, J., Cluster driven anisotropic diffusion for speckle reduction in ultrasound images, In IEEE International Conference on Image Processing (ICIP) (pp. 2325-2329), 2016.

[45] Mishra, D., Chaudhury, S., Sarkar, M., Soin, A. S., \& Sharma, V. (2017), Edge probability and pixel relativity-based speckle reducing anisotropic diffusion, IEEE Transactions on Image Processing, 27(2):649-664, 2017.

[46] Zhou, Z., Guo, Z., Zhang, D., \& Wu, B., A nonlinear diffusion equation-based model for ultrasound speckle noise removal, Journal of Nonlinear Science, 28(2): 443-470, 2018.

[47] Gao, M., Kang, B., Feng, X., Zhang, W., \& Zhang, W., Anisotropic Diffusion Based Multiplicative Speckle Noise Removal, Sensors, 19(14): 31543164, 2019.

[48] Xu, H. H., Gong, Y. C., Xia, X. Y., Li, D., Yan, Z. Z., Shi, J., \& Zhang, Q., Gabor-based anisotropic diffusion with lattice Boltzmann method for medical ultrasound Despeckling, Mathematical Biosciences and Engineering, 16(6): 7546-7561, 2019.

[49] Goyal, S., Rani, A., Yadav, N., \& Singh, V., SGSSRAD Filter for De-noising and Edge Preservation of Ultrasound Images, In 6th International Conference on Signal Processing and Integrated Networks (SPIN), (pp. 676-682), 2019.

[50] Wang, Y., Zhang, L., \& Li, P., Local variancecontrolled forward-and-backward diffusion for image enhancement and noise reduction, IEEE Transactions on Image Processing, 16(7): 1854-1864, 2007.

[51] Chao, S. M., \& Tsai, D. M., An anisotropic diffusion-based defect detection for low-contrast glass substrates, Image and Vision Computing, 26(2): 187-200, 2008.

[52] Chao, S. M., \& Tsai, D. M., An improved anisotropic diffusion model for detail-and edge-preserving smoothing, Pattern Recognition Letters, 31(13): 2012-2023, 2010.

[53] Chao, S. M., \& Tsai, D. M., Anisotropic diffusion with generalized diffusion coefficient function for defect detection in low-contrast surface images, Pattern Recognition, 43(5): 1917-1931, 2010.

[54] Khan, U. N., Arya, K. V., \&Pattanaik, M., Histogram statistics based variance controlled adaptive threshold in anisotropic diffusion for low contrast image enhancement, Signal Processing, 93(6): 1684-1693, 2013.

[55] Ham, B., Min, D., \&Sohn, K., Revisiting the relation-ship between adaptive smoothing and anisotropic diffusion with modified filters, IEEE transactions on Image Processing, 22(3): 1096-1107, 2013.

[56] Weickert, J., Multiscale texture enhancement, International Conference on Computer Analysis of Images and Patterns (pp. 230-237). Springer, Berlin, Heidelberg, 1995. 
[57] Zhou, Q., Gao, J., Wang, Z., \& Li, K., Adaptive variable time fractional anisotropic diffusion filtering for seismic data noise attenuation, IEEE Transactions on Geoscience and Remote Sensing, 54(4): 1905-1917, 2015.

[58] Ben Gharsallah, M., Ben Mohammed, I., \& Ben Braiek, E., Improved Geometric Anisotropic Diffusion Filter for Radiography Image Enhancement, Intelligent Automation \& Soft Computing, 1-9, 2016.

[59] Chen, Y., Bai, Y., Zhang, Q., Wang, Y., \&Gui, Z., Self-Adaptive Anisotropic Image Enhancement Algorithm Based on Local Variance,Journal of Engineering Science \& Technology Review, 10(3), 2017

[60] Liu, H., \& Zhang, J., Filtering combined dynamic stochastic resonance for enhancement of dark and low-contrast images, International Conference on Progress in Informatics and Computing (PIC), pp. 133-137, 2017.

[61] Cho, S. I., \& Kang, S. J., Geodesic path-based diffusion acceleration for image denoising, IEEE Transactions on Multimedia, 20(7): 1738-1750, 2018.

[62] Chen, C. S., Weng, C. M., \& Tseng, C. C., An efficient detection algorithm based on anisotropic diffusion for low-contrast defects, The International Journal of Advanced Manufacturing Technology, 94(9-12): 4427-4449, 2018.

[63] Nair, R. R., David, E., \&Rajagopal, S., A robust anisotropic diffusion filter with low arithmetic complexity for images, EURASIP Journal on Image and Video Processing, 48(1), 2019.

[64] Chen, Q., Liu, B., \& Zhou, F., Anisotropy-based image smoothing via deep neural network training, Electronics Letters, 55(24): 1279-1281, 2019.

[65] Eskicioglu, A. M., \& Fisher, P. S., Image quality measures and their performance, IEEE Transac-tions on Communications, 43(12): 2959-2965, 1995.

[66] Larson, E. C., \& Chandler, D. M., Most apparent distortion: full-reference image quality assessment and the role of strategy, Journal of Electronic Imaging, 19(1): 006-011, 2010.

[67] Lin, W., \&Kuo, C. C. J., Perceptual visual quality metrics: A survey, Journal of Visual Communication and Image Representation, 22(4): 297-312, 2011.

[68] Pratt, W. K., \& Wiley, J., A Wiley-Interscience Publication. In Digital Image Processing, 1978.

[69] Narvekar, N. D., \&Karam, L. J., A no-reference image blur metric based on the cumulative probability of blur detection (CPBD), IEEE Transactions on Image Processing, 20(9): 2678-2683, 2011.

[70] Choi, L. K., You, J., \&Bovik, A. C., Referenceless prediction of perceptual fog density and perceptual image defogging, IEEE Transactions on Image Processing, 24(11): 3888-3901, 2015.

[71] Wang, Z.,\&Bovik, A. C., A universal image quality index, IEEE Signal Processing Letters, 9(3): 81-84, 2002.

[72] Wang, Z., Bovik, A. C., Sheikh, H. R., \&Simoncelli, E. P., Image quality assessment: from error visibility to structural similarity, IEEE Transactions on Image Processing, 13(4): 600-612, 2004.

[73] Brooks, A. C., Zhao, X., \& Pappas, T. N., Structural similarity quality metrics in a coding context: Exploring the space of realistic distortions, IEEE Transactions on Image Processing, 17(8): 12611273, 2008.

[74] Wang, Z., \&Bovik, A. C., Mean squared error: Love it or leave it? A new look at signal fidelity measures, IEEE Signal Processing Magazine, 26(1): 98-117, 2009.

[75] Ding, L., Huang, H., \&Zang, Y., Image quality assessment using directional anisotropy structure measurement, IEEE Transactions on Image Processing, 26(4): 1799-1809, 2017.

[76] Sheikh, H. R., LIVE image quality assessment database release $2 . \quad$ http://live.ece.utexas. edu/research/quality, 2005.

[77] Ponomarenko, N., Lukin, V., Zelensky, A., Egiazarian, K., Carli, M., \&Battisti, F., TID2008-a database for evaluation of full-reference visual quality assessment metrics, Advances of Modern Radioelectronics, 10(4): 30-45, 2009.

[78] Wernick, M. N., Yang, Y., Brankov, J. G., Yourganov, G., \&Strother, S. C., Machine learning in medical imaging, IEEE Signal Processing Magazine, 27(4): 25-38, 2010.

[79] Talebi, H., \&Milanfar, P., NIMA: Neural image assessment, IEEE Transactions on Image Processing, 27(8): 3998-4011, 2018

[80] Khan, U. N., Arya, K. V., \&Pattanaik, M., Fuzzy based Diffusion Coefficient Function in Anisotropic Diffusion for Impulse Noise Removal. In Eighth Indian Conference on Computer Vision, Graphics and Image Processing (ICVGIP 2012), December 16 19, IIT Bombay, India, 2012.

[81] Succi, Sauro, The Lattice Boltzmann Equation for Fluid Dynamics and Beyond. Oxford University Press. ISBN 978-0-19-850398-9, 2001.

[82] Savitzky, A.; Golay, M.J.E., Smoothing and Differentiation of Data by Simplified Least Squares Procedures, Analytical Chemistry, 36 (8): 1627-39, 1964.

[83] Khan, U. N., Arya, K. V., \&Pattanaik, M., Edge Preservation of Impulse Noise Filtered Images by Improved Anisotropic Diffusion, Multimedia Tools and Application, Vol. 73: 573 - 597, 2013.

[84] Dang Thanh, Prasath Surya, Le Minh Hieu, A Review on CT and X-Ray Images Denoising Methods, Informatica, 43 (2): 151 - 159, 2019.

[85] Nguyen Chi Sy, Ha Hoang Kha and Nguyen Minh Hoang, A Robust Image Watermarking Scheme Based on the Laplacian Pyramid Transform, Informatica, 44(1): 75 - 84, 2020.

[86] Nguyen Hoang Hai, Le Minh Hieu, Dang N. H. Thanh, Nguyen Van Son and V. B. Surya Prasath, An Adaptive Image Inpainting Method Based on the Weighted Mean, Informatica, 43(4): 507 - 513, 2019. 